\title{
A Coupled Approach for Structural Damage Detection With Incomplete Measurements
}

\author{
George James, Tim Cao, Mo Kaouk \\ Loads and Structural Dynamics Branch \\ NASA Johnson Space Center \\ Houston, Texas 77058 \\ David Zimmerman \\ University of Houston \\ Houston, Texas 77204
}

\begin{abstract}
This historical work couples model order reduction, damage detection, dynamic residual/mode shape expansion, and damage extent estimation to overcome the incomplete measurements problem by using an appropriate undamaged structural model. A contribution of this work is the development of a process to estimate the full dynamic residuals using the columns of a spring connectivity matrix obtained by disassembling the structural stiffness matrix. Another contribution is the extension of an eigenvector filtering procedure to produce full-order mode shapes that more closely match the measured active partition of the mode shapes using a set of modified Ritz vectors. The full dynamic residuals and full mode shapes are used as inputs to the minimum rank perturbation theory to provide an estimate of damage location and extent. The issues associated with this process are also discussed as drivers of near-term development activities to understand and improve this approach.
\end{abstract}

\section{KEYWORDS}

Damage Detection, Structural Health Monitoring, Model Order Reduction, Mode Shape Expansion, Ritz Vectors

\section{INTRODUCTION}

The use of changing structural dynamics parameters as indicators of damage state and structural health has received increasing attention in recent years. The global nature of these parameters provides a unique capability to monitor internal components of aerospace, civil, mechanical, naval, and nuclear structures. The ability to model the structural response analytically enhances this unique capability to locate damage in unmeasured locations as well as to provide tools to determine the extent of the damage. However, much work needs to be done to use experimental and analytical models properly in a coordinated fashion to perform damage identification. One of the most difficult issues to overcome is the incomplete measurement problem, which means that 1) there is an inherent mismatch between the experimental and the analytical degrees of freedom (DOFs) and 2) there is a mismatch between the numbers of measured and analytical modes. Another difficult issue is that neither the experimental measurements (noise) nor the analytical model (modeling errors) is correct. The last statement is especially true in damage identification as the analytical model is typically produced for the undamaged structure.

The incomplete measurement problem means that model order reduction [1-6] and/or test data expansion [4-11] must be performed to utilize both data sets fully. However, it has been found that most reduction expansion techniques are not extremely useful for damage identification [6, 10, 11]. It has been suggested that reduction/damage identification/expansion should be handled as coupled problems [6]. This work describes the development of such an integrated procedure that is initiated by first disassembling the structural stiffness matrix [12-15] into a connected set of springs. The columns of the spring connectivity matrix are then used as a set of candidate basis vectors for estimating the full dynamic residual. This candidate set is down selected using the modal assurance criteria (MAC) between the reduced dynamic residual and a reduction of each candidate vector averaged over the modes of interest. A least-squares fit is then 
used to scale the remaining basis vectors to match the dynamically reduced residuals for each mode. The estimated full residuals provide damage location information and are used to initiate a modified Ritz vector [16] calculation process. A set of Ritz-like vectors are calculated and used as a set of basis vectors to expand the mode shapes and to guarantee mass orthogonality. The full dynamic residuals and full mode shapes are then used as inputs to the minimum rank perturbation theory (MRPT) [17-19] to provide an estimate of damage. The process suggested herein was originally published in references $[20,21]$.

\section{TECHNICAL BACKGROUND OF ORIGINAL WORK}

This section briefly describes the basic theoretical components used in the coupled damage detection approach.

\section{Dynamic Residual Formulation}

This study will proceed with a standard math model for a dynamical system (ignoring damping):

$$
[M]\{\ddot{U}\}+[K]\{U\}=\{F\} \text {. }
$$

The corresponding eigenvalue problem for the undamaged structure is given as:

$$
\left.\left\lfloor M \omega_{u i}^{2}-K\right\rfloor \phi_{u i}\right\}=0
$$

where the subscript $u$ denotes undamaged and $i$ denotes the $i$ th mode of vibration. The eigenvalue problem for the damaged structure is:

$$
\left.\left\lfloor(M-\Delta M) \omega_{i}^{2}+(K-\Delta K)\right\rfloor \phi_{i}\right\}=0
$$

where the $\Delta$ matrices represent the effect of damage on the structural property matrices. Now Eq. (3) can be rewritten in dynamic residual form:

$$
\left.\left\lfloor M \omega_{i}^{2}+K\right\rfloor\left\{\phi_{i}\right\}=\{b\}=\left\lfloor\Delta M \omega_{i}^{2}+\Delta K\right\rfloor \phi_{i}\right\}
$$

\section{Model Reduction}

The dynamic residual form can be rearranged into an active or measured set of DOFs and an omitted or unmeasured set of DOFs. It is commonly assumed that the model of the undamaged structure can be used to create a transformation relating the unmeasured and measured DOFs. One approach is to produce a physical-coordinates based transformation [7] that for a single mode shape has the following form:

$$
\left\{\phi_{i}\right\}=\left\{\begin{array}{l}
\phi_{a i} \\
\phi_{o i}
\end{array}\right\}=[T]\left\{\phi_{a i}\right\}=\left[\begin{array}{c}
T_{a a} \\
T_{o a}
\end{array}\right]\left\{\phi_{a i}\right\}
$$

The transformation matrix $[T]$ in Eq. (5) can be calculated from the stiffness matrix $[K]$ using the static or Guyan-Irons $[1,2]$ reduction/expansion. A scaled sum of the mass $[M]$ and stiffness matrices can also be used to estimate $[T]$ as suggested by dynamic reduction/expansion [3]. The transformation matrix [T] denoted in Eq. (5) can be used to reduce the order of the analytical model. Substituting Eq. (5) into Eq. (4) and premultiplying by $[T]^{T}$, one finds that the reduced equations of motion are:

$$
[T]^{T}\left[M \varpi_{i}^{2}+K\right][T]\left\{\phi_{a i}\right\}=\left\{\boldsymbol{b}_{r i}\right\}=[T]^{T}\left\{b_{i}\right\}
$$

The reduced dynamic residual, $\left\{b r_{i}\right\}$, given in Eq. (6) has been shown to be of importance in damage identification [1722] to localize and to calculate the extent of damage. However, these capabilities are limited by the application of the 
transformation $[T]$, which tends to redistribute residual forces to undamaged DOFs. A primary contribution of this work is to provide an approach to retain these capabilities by first estimating the full dynamic residual $\left\{b_{i}\right\}$. The procedure suggested to perform this estimation begins by performing a matrix disassembly on the stiffness matrix [12-15]. This will produce a set of basis vectors used to estimate the full dynamic residual.

\section{Matrix Disassembly}

Matrix disassembly is a process that decomposes a structural matrix into a matrix representation of the connectivity between DOFs and a matrix containing the magnitude information [12-15]. This formulation has the following form for the stiffness matrix:

$$
[K]=\left[C P C^{T}\right]
$$

Advanced applications utilize a disassembly into the same finite elements that were used to create the model [14]. However, this work utilizes a disassembly into a set of equivalent springs. This produces a general technique that can be applied to any model without detailed knowledge about the actual elements used in the assembly. The matrix $[C]$ is an $n x$ $m$ matrix, where $n$ is the matrix dimension for $[K]$ and $m$ is equal to the total number of unique entries in $[K]$ (for symmetric $[K]$ this amounts to the nonzero entries in the upper triangular portion of the stiffness matrix). The diagonal matrix $[P]$ is calculated as:

$$
\begin{aligned}
& P(i, i)=\sum_{j=1}^{n} K(i, j), \text { for } \mathrm{i}=1: \mathrm{n} ; \text { and } \\
& P(i, i)=-K(j, k), \text { for } \mathrm{i}=\mathrm{n}+1: \mathrm{m} .
\end{aligned}
$$

The first $n$ columns/rows of [C] form an $n \times \mathrm{x} n$ identity matrix. The remaining $(m-n)$ columns are defined according to the element locations of the unique entries in the matrix $[K]$. For the element $K(j, k)$, which is used to define the $i$ th row/column of $[P]$ for the index $i$ running from $n+1$ to $m$, the $i$ th column of $[C]$ is given as:

$$
\begin{gathered}
C(j, i)=1.0 ; \text { and } \\
C(k . i)=-1.0 .
\end{gathered}
$$

\section{Estimating the Expanded Dynamic Residual}

For each mode of interest, equation (6) relates a known quantity ( $\left\{b_{r i}\right\}$, the reduced dynamic residual) to an unknown quantity $\left(\left\{b_{i}\right\}\right.$, the full dynamic residual). This work assumes that the full dynamic residual can be described as a linear combination of the columns of the connectivity matrix. Therefore, each column will initially be considered a candidate for inclusion in the calculation of the full dynamic residual vector for that mode. The first issue is to down select the set of columns of $[C]$ to the most promising subset for further analysis. Although it was not rigorously necessary, this work constrained the selected subset to have linearly independent columns. (The full $[C]$ matrix contains a high degree of column linear dependence.) Each candidate vector from $[C],\left\{c_{j}\right\}$, will serve as an approximate full dynamic residual vector. The resulting reduced dynamic residual basis vector $\left\{\beta_{r i j}\right\}$ is calculated from equation (6) by replacing $\left\{b_{i}\right\}$ with $\left\{c_{j}\right\}$. In this work, a comparison with the actual reduced dynamic residual for that mode is made using the MAC, which relates the global similarity between two vectors:

$$
M A C_{i j}=\frac{\left\|b_{r i}^{T} \beta_{r i j}\right\|^{2}}{\left\|b_{r i}^{T} b_{r i}\right\|\left\|\beta_{r i j}^{T} \beta_{r i j}\right\|} .
$$

The MAC values are averaged over the modes of interest. These averaged values then provide a discriminator between the candidate columns of the connectivity matrix. Columns $\left\{c_{j}\right\}$, which produce large MAC values over a wide range of modes, are candidates for down selection. The user must select the number of the largest average MAC candidate basis 
vectors to include in the next analysis. This selection is made based on the relative sizes of the average MAC values, the spatial relationship between the candidate vectors, and the linear dependence of the resulting set of vectors.

The next step is to use the selected vectors as basis vectors for approximating the full dynamic residual. Substituting a linear combination of the basis vectors into equation (6) yields:

$$
\left\{\boldsymbol{b}_{r i}\right\}=[T]^{T}\left\{\boldsymbol{b}_{i}\right\}=[T]^{T}\left[\boldsymbol{C}_{\text {selected columns }}\right]\left\{\alpha_{i}\right\}
$$

where the unknown coefficient $\alpha_{i}$ is determined by a least-squares approach. (Note that the reduction transformation will change with each mode if dynamic reduction is used.) The critical output of this step is a set of full dynamic residual vectors that match the reduced equations. The next step will be to use these expanded residuals to expand the mode shapes.

\section{Mode Shape Expansion}

Mode shape expansion estimates the unmeasured DOFs given the undamaged model, the experimental modal frequency, the measured mode shape DOFs, and the full dynamic residual. However, the physical coordinate transformation approach listed earlier typically assumes that the omitted partition of the dynamic residual is zero [1-3]. Because this may not be the case for the damage identification problem, this approach must be modified assuming a nonzero residual vector.

An approach called eigenvalue filtering (EV) utilizes the full residual to expand the mode shape. This approach uses eqution (4) to generate the expansion by inverting the dynamic stiffness matrix $[D]$ using the associated experimental modal frequency $\omega_{i}$ (reference [23]):

$$
\left\{\phi_{i}\right\}=\left[M \omega_{i}^{2}+K\right]^{-1}\left\{b_{i}\right\}=\left[D_{i}\right]^{-1}\left\{b_{i}\right\}
$$

The EV approach ensures that the full mode shape produces the estimated dynamic residual when operating on the undamaged model shifted with the experimental modal frequency. However, it should be noted that the active partitions of the mode shapes are not constrained to be the measured values. One contribution of this work is to extend this approach to allow the user to add in these constraints in an incremental process.

The modified approach assumes that the full mode shapes are a linear combination of basis vectors. A modified Ritz vector calculation will be used to generate a set of basis vectors to expand the mode [16]. The Ritz vector calculation procedure provided in reference [16] initiates the process by operating on a force vector with the inverse of the stiffness matrix. The resulting deflection vector is mass normalized and used as the input vector to an inverse iteration with the stiffness matrix inverse and mass matrix as the multipliers. The successive Ritz vectors are orthogonalized with a modified Gram-Schmidt process and mass normalized.

The modified process will generate a set of Ritz-like vectors for each mode of interest using the dynamic residual for each mode as the initial force vector. Two additional modifications to the Ritz vector calculation approach referenced earlier are required. First, to be consistent for perfect data and perfect dynamic residuals, the stiffness matrix is replaced with the dynamic stiffness matrix $[D]$ appropriate for each mode. The next modification is that the mass orthogonalization step includes not just the previously calculated Ritz vectors for that mode but also the previously expanded mode shapes. Hence, a set of basis vectors responding to the forces represented by the dynamic residual and mass orthogonal to themselves and the previously expanded modes of the system will be calculated. Therefore, a linear combination of these basis vectors will be mass orthogonal to the previously expanded modes of the system. These basis vectors will be linearly combined to match the active partition of the mode of interest. Hence for the $l$ th mode of the system the following equations are used:

$$
\left\{\bar{v}_{1}\right\}=\left[D_{l}\right]^{-1}\left\{b_{l}\right\}
$$




$$
\begin{aligned}
& \left\{\boldsymbol{V}_{1}\right\}=\frac{\left\{\overline{\boldsymbol{V}}_{1}\right\}}{\left(\overline{\boldsymbol{V}}_{1}^{T} M \overline{\boldsymbol{V}}_{1}\right)^{1 / 2}} \\
& \left\{\overline{\boldsymbol{\nu}}_{i}\right\}=\left[\boldsymbol{D}_{l}\right]^{-1}[M]\left\{\boldsymbol{V}_{i-1}\right\}, \\
& \left\{\boldsymbol{V}_{i}\right\}=\left\{\overline{\boldsymbol{V}}_{i}\right\}-\sum_{j=1}^{i-1}\left(\boldsymbol{V}_{j}^{T} M \overline{\boldsymbol{V}}_{i}\right)\left\{\boldsymbol{V}_{j}\right\}-\sum_{k=1}^{l-1}\left(\boldsymbol{\phi}_{k}^{T} M \overline{\boldsymbol{V}}_{i}\right)\left\{\boldsymbol{\phi}_{k}\right\} \\
& \left(\boldsymbol{V}_{i}^{T} M \boldsymbol{V}_{i}\right)=1 \\
& \left\{\phi_{l}\right\}=\sum_{i=1}^{n} \gamma_{l i}\left\{\boldsymbol{V}_{i}\right\}
\end{aligned}
$$

This procedure produces a set of expanded mode shapes that are mass orthogonal and consistent with the full dynamic residuals. It is important to note that the first vector is equivalent to the full mode shape calculated with the EV approach given in equation (12). The coefficients $\gamma_{l i}$ in equation (18) are determined by partitioning equation (18) into the active and omitted sets and then solving a least-squares problem involving only the active set. Hence, by allowing additional terms in the expansion process, the user can trade off confidence in the expanded dynamic residual for confidence in the measured mode shape DOFs.

\section{Damage Location and Extent: MRPT}

The estimation of damage location and extent is performed via the Minimum Rank Perturbation Theory (MRPT) [17-20]. The underlying philosophy of the MRPT is that reduced rank perturbations to the structural matrices are the manifestation of damage. In this approach, damage results in a zero-nonzero pattern in the dynamic residual as given by equation (4). Typically, measurement noise and model order reduction or expansion destroys this pattern. Hence, a significant result of this approach is that the zero-nonzero pattern of the dynamic residual will be controlled by the columns of the connectivity matrix that are used to create it.

The estimation of damage extent using a minimum rank formulation utilizes the following calculation (assuming that all damage is manifested only in the stiffness matrix):

$$
[\Delta K]=[B]\left[\Phi^{T} B\right]^{-1}\left[B^{T}\right]
$$

where $[B]$ is the matrix formed of all column vectors of the expanded dynamic residuals for the modes of interest and [ $\Phi]$ is the matrix of the expanded modal vectors.

\section{EXAMPLE APPLICATION OF ORIGINAL WORK}

The example application chosen for this section closely parallels the examples provided in precursory work to provide a context for interpreting the results $[6,11]$.

\section{NASA Eight-Bay Truss}

For the comparisons in this work the NASA eight-bay truss structure [24] is utilized. The eight-bay truss structure is an experimental test article developed to study a variety of damage identification issues. The cantilevered truss includes 32 nodes, each of which was instrumented with triaxial accelerometers. Fifteen unique damage cases were produced by removing individual truss members. The structure and representations of the damage cases (denoted by alphabetic characters) are provided in Figure 1. This work uses 12 modes from a 96-DOF analytical model of the undamaged structure and the damage cases to explore the developments discussed in previous sections. The DOFs that are assumed to be active or measured for the example provided in this section are denoted by circles in Figure 1. At these nodes, full triaxial meas- 
urements are utilized, resulting in a total measurement set of 24 DOFs. Case D will be used for the examples provided in this section.

\section{Spring Disassembly}

The structural matrices for this model include 9216 (96x96) potential entries. However, there are only 320 unique (excluding symmetric entries) nonzero entries in this matrix. After reordering the matrices into analysis and omitted DOFs, the 224 off-diagonal elements are disassembled into 224 springs between DOFs. The 96 diagonal entries are disassembled as springs to ground. Note that this disassembly process does not disallow negative spring stiffness as these springs are usually a simplified representation of a more complicated structural element. It is also important to note that this connectivity pattern becomes an inherent part of subsequent estimations of full residuals, damage location, expanded mode shapes, and damage extent using the procedure outlined in the preceding section.

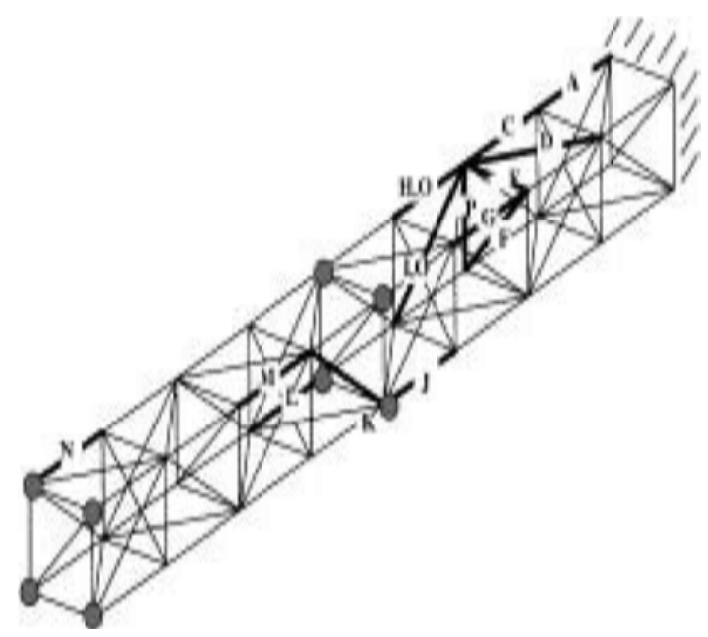

Figure 1. NASA Eight-Bay Truss Structure.

\section{Selection of Residual Basis Functions}

The example presented herein ignored the 96 springs to ground representing the diagonal elements. This enhanced the column linear independence of the down-selected subset. Of the remaining 244 possible basis vectors, fewer than 20 had average MAC values over 0.4. Of these, the six with the highest averaged MAC values were selected. The general rule used to date is to select a number equal to half of the number of modes of interest unless a break in the connectivity of the elements or a rank deficiency (column linear dependence) sets in. Figure 2 provides plots of the six candidate basis functions chosen. These functions do seem to be targeting certain locations on the structure. In fact, the affected DOFs of damage case $\mathrm{D}(69,70,86$, and 87$)$ are well represented in these vectors. The first, third, and sixth vectors exclusively relate to these affected DOFs.

\section{Dynamic Residual Reduction/Expansion}

The coefficients for the fit of the six basis vectors shown in Figure 2 to the first dynamic residual using equation (11) are $-.7328,0,-.7328,0,0$, and 1.4657 . Hence, the first, third, and sixth basis vectors are pulled out as the true components of the full dynamic residual, as indicated in Figure 3. The true and calculated full residuals for the first mode are given in the upper plots of Figure 3. The true and calculated reduced residuals for the first mode are provided in the lower plots. It is obvious that the correct residuals have been obtained in both cases. However, a more important observation is that the reduced residuals do not maintain the location information in a form that is easily or visually interpreted. Hence, the primary thrust of this work (the estimation of the full residuals) is strongly suggested as important to advancing the field. 
Mode Shape Expansion

For the example provided in this section,12 Ritz-like basis vectors were calculated using equations (13-18). The fact that noise-free measurements are being utilized produces a situation where the first basis vector exactly matches the expanded mode shape. Figure 4 supports these results for the first mode. The expanded and true mode shapes are provided in the upper plots and are seen to be equivalent. The first and second basis vectors are provided in the lower plots. It is obvious that the first basis vector captures the first expanded mode shape. This important point can be used to monitor the consistency of a data set and/or the selection of dynamic residual basis vectors.
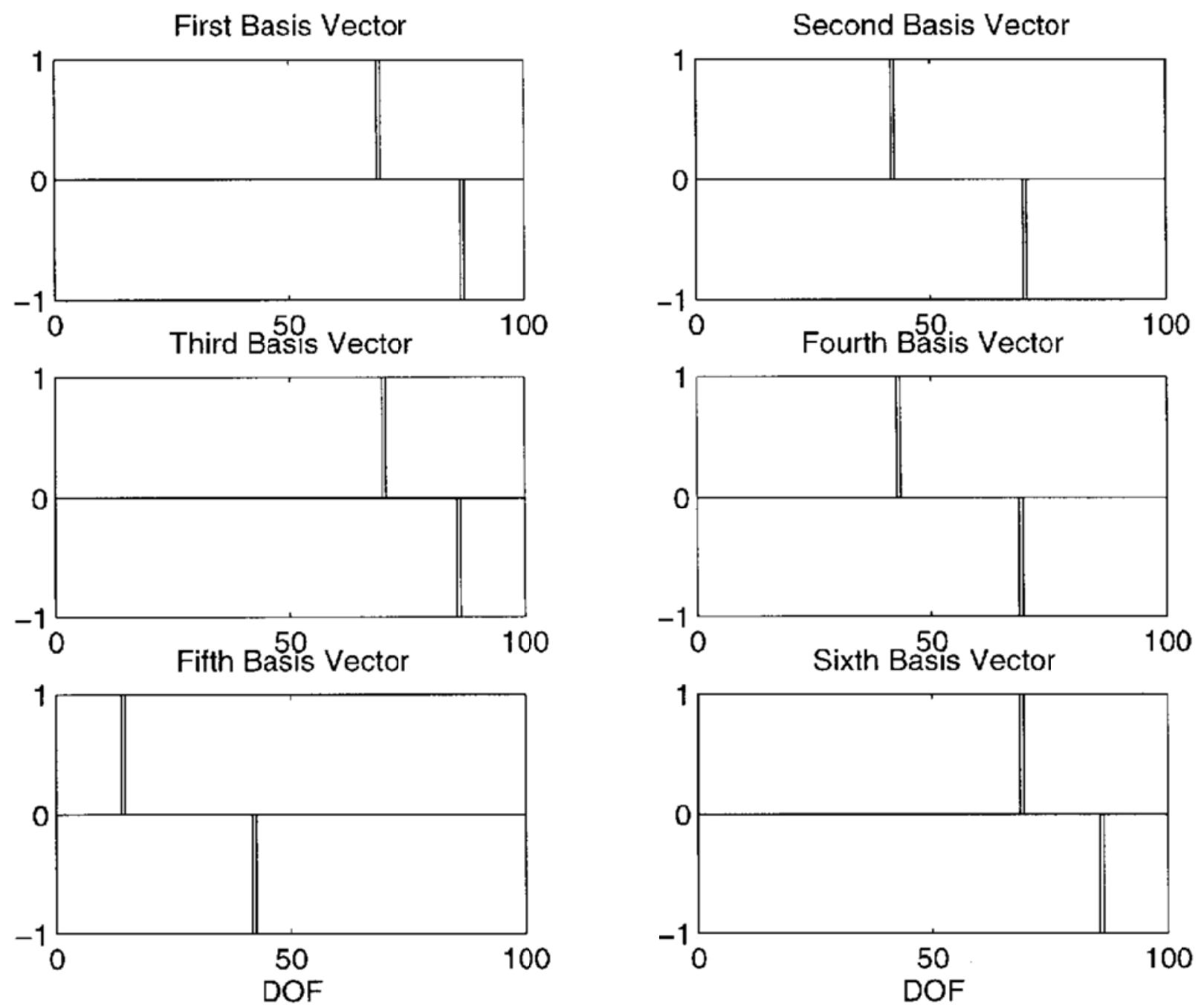

Figure 2. Six Selected Basis Functions.

Damage Extent

The MRPT update is then calculated using the expanded mode shapes and full dynamic residuals. The upper-left-hand plot of Figure 5 shows the absolute value of this update. The upper-right-hand plot shows the true update, which suggests that the update is very close. In fact, the lower-left-hand plot provides the error or difference between the two $[\Delta K]$ matrices. The magnitudes of the differences are at least two orders of magnitude less than the true values. The updated stiffness matrix is provided in the lower-right-hand plot as a point of reference. Besides providing an example of the process developed earlier, this example shows that the process does provide the correct results for perfectly measured data. However, the selection of dynamic residual basis vectors is critical. 

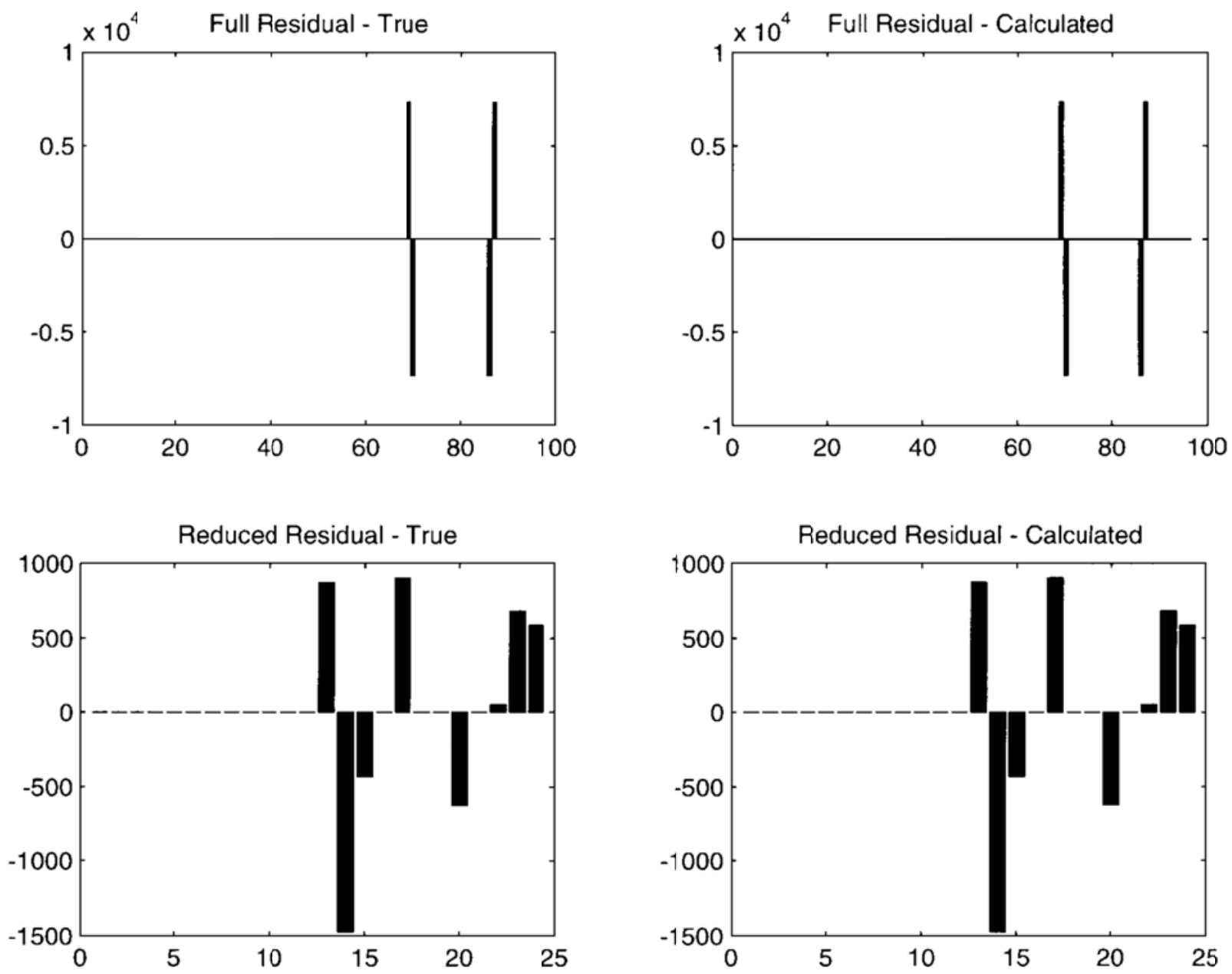

Figure 3. First Mode Dynamic Residuals.

\section{APPLICATION OF ORIGINAL METHOD TO EXPERIMENTAL DATA}

The coupled damage identification procedure developed earlier was applied to the experimental data for damage case D of the NASA eight-bay truss structure. The data contained five modes with 96 measurement locations as listed earlier. However, only the 24 active DOFs shown in Figure 1 were used in this example. This represents one of the most difficult active sensor sets due to the lack of even spatial distribution. It was found that not all modes contributed to the selection of the dynamic residual basis vectors. Modes 4 and 5 had almost no ability to discriminate between the vectors. This may be due in part to the analytical model used in the experimental study. The stiffness matrix was fully populated as a result of the update procedure required to.$t$ the experimental undamaged data. The original sparse stiffness matrix for this system did show these modes as having the ability to discriminate between the vectors. However, the inability of this model to match the undamaged data precluded its use in this study.

Table 1 lists the order of appearance of each of the primary vectors, as determined from the analytical study (196, 197, and 198), as a function of the number of experimental modes used. Vector 198 is consistently high (place 1 or 2). Vector 197 is much lower than in the analytical studies but still reasonably high. Vector 196 was outside the range of reasonable orders to consider. 
First Mode Shape - Expanded

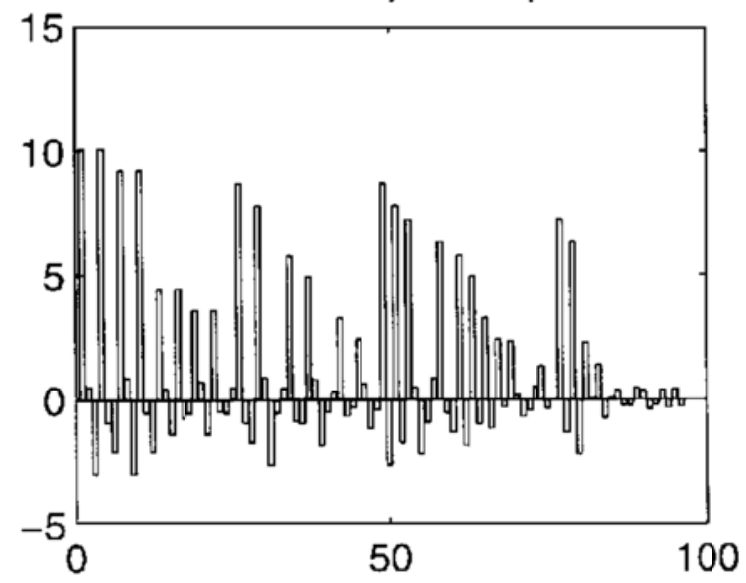

First Ritz Vector

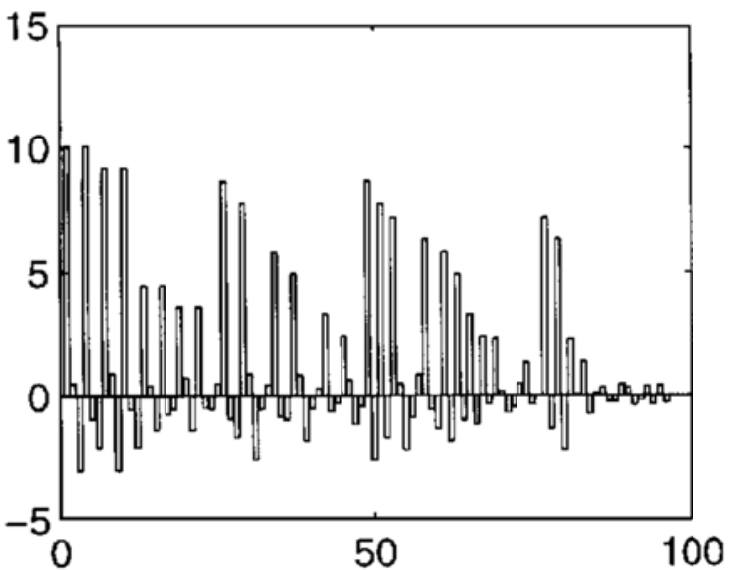

First Mode Shape - True
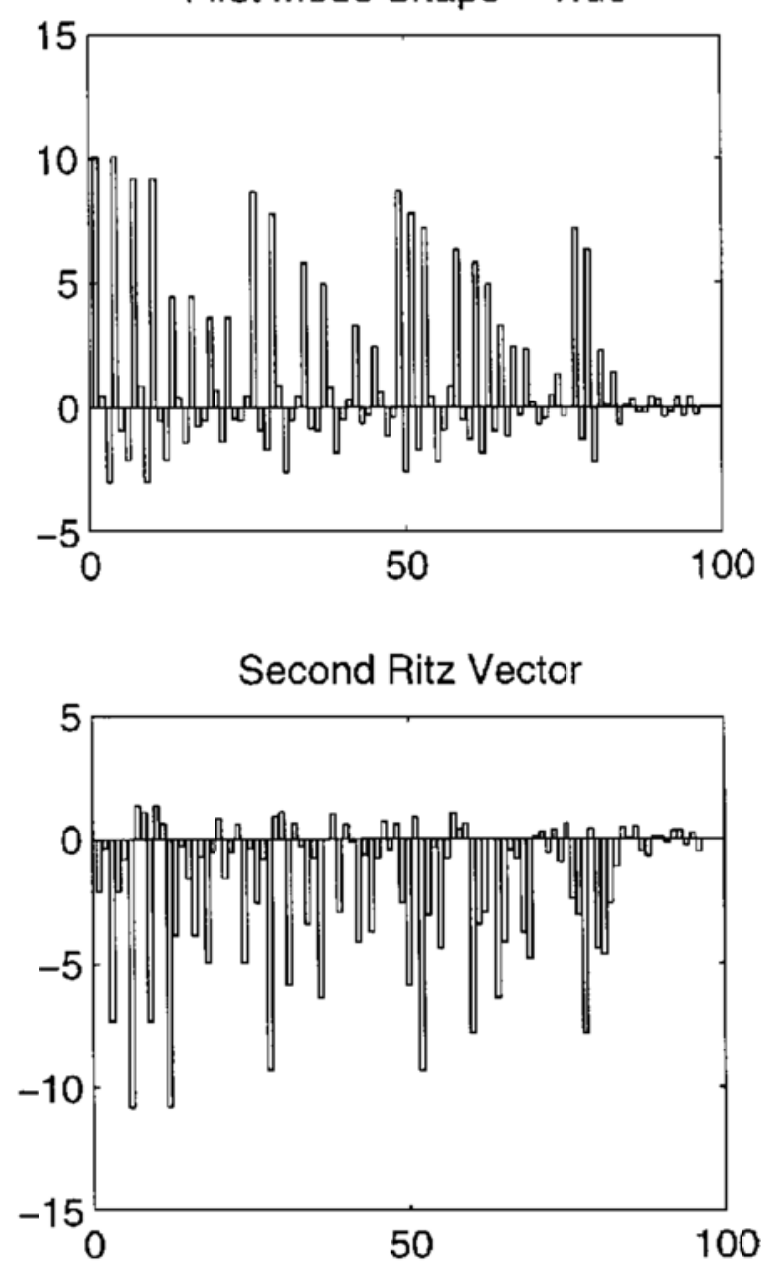

Figure 4. First Expanded Mode Shape.

Figure 6 provides plots of four different manifestations of the model update matrices. The upper-left-hand plot is the analytical update matrix that provides the best estimate of what the experimental results should produce. The upper-right hand plot provides what could be considered the best estimate of the update matrix if the user knew the results of the previous analytical study and the damage location. For this estimation, the first two modes and basis vectors 198, 197, and 196 were used. The magnitudes are about half of the expected magnitudes, and the sparsity is reasonable. Note that two Ritz-like basis vectors were used to expand the modes. The lower-left-hand plot contains what might be considered a reasonable update for a user with no knowledge of the damage location but plenty of time to work with the data and to develop the proper insight. For this update, the first two modes were used to select the residual basis vectors, and only one basis vector was used-vector 198. The lower-right-hand plot represents a blind application of the process. All five modes were used, and the first six residual basis vectors were selected. Changes were seen at many other locations in the structure, however the region of the model affected by the damage is dense with large changes. The magnitudes of the changes were roughly equivalent to the other two experimental updates. 

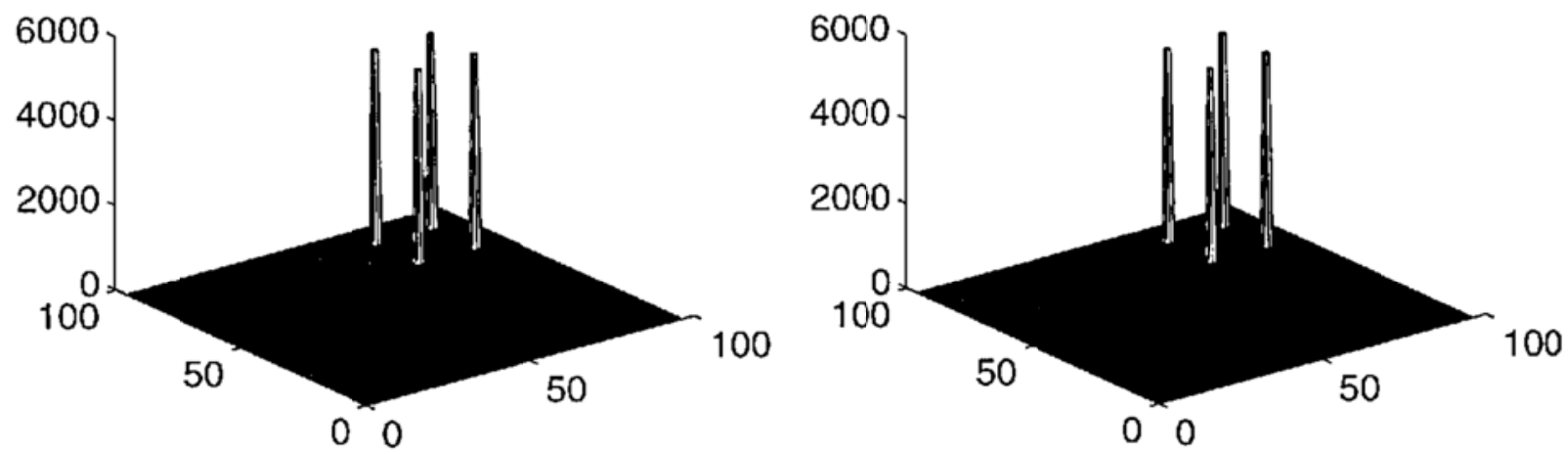

\section{Error Matrix for DeltaK (Absolute Values)}

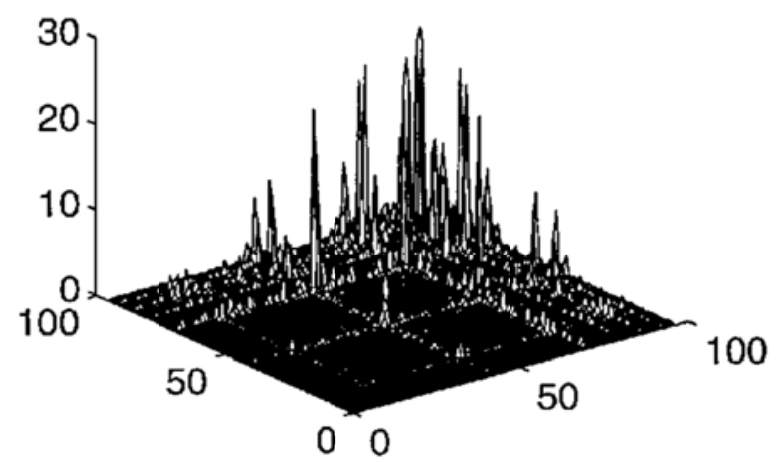
Updated Stiffness Matrix (Absolute Values) $\times 10^{4}$

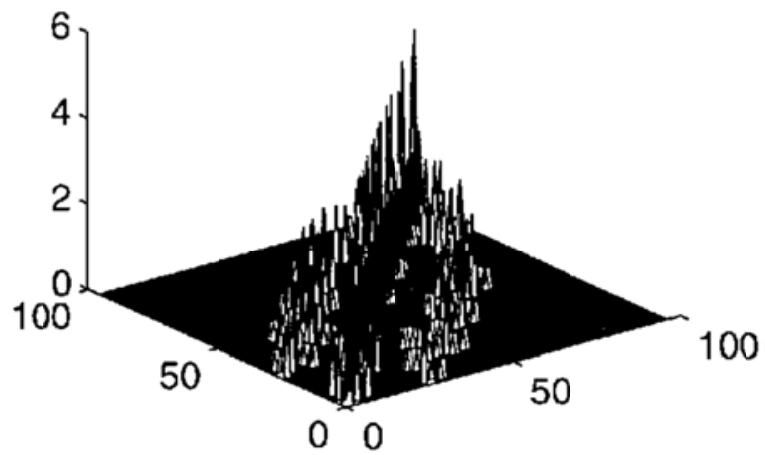

Figure 5. Damage Extent Calculation.

\section{ASSESSMENT OF ISSUES WITH ORIGINAL WORK}

The original work $[20,21]$ studied the sensitivity of the original procedure to three implementation issues including: (a) the number of residual basis vectors selected, (b) the number of modes used, and (c) the noise in the measurements. The previous studies suggested that additional work was needed to develop experience and techniques for sorting and choosing residual basis vectors. Additionally, the previous efforts suggested that there existed a minimum number of modes to discriminate between the residual basis vectors. And finally, the original process appears to be fairly robust with respect to noise based on the limited studies performed previously. In actuality, these issues are a subset of technical issues that need to be systematically assessed and mitigated if possible with this coupled damage detection approach. Figure 7 suggests a more complete set of technical issues that are associated with each step in the original process.

\section{Model of Undamaged Structure}

The primary issue with this step is the need for the original undamaged model to correlate with the actual structure of interest. It can be assumed that the technical errors associated with this step may be reduced or eliminated for some measured modes if a model updating step is performed using undamaged data from the structure. 


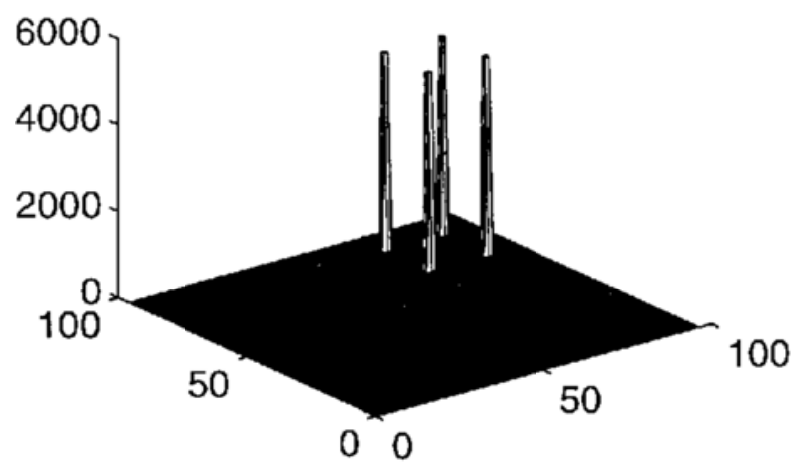

\section{DeltaK - Reasonable (Absolute Values)}

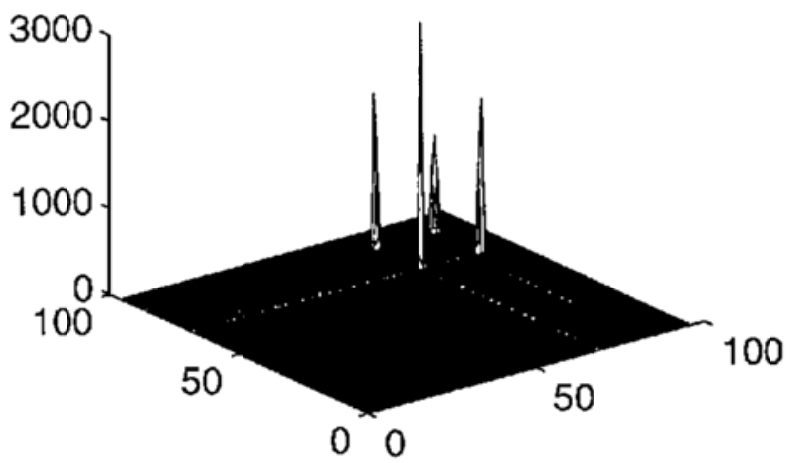

\section{DeltaK - Blind (Absolute Values)}
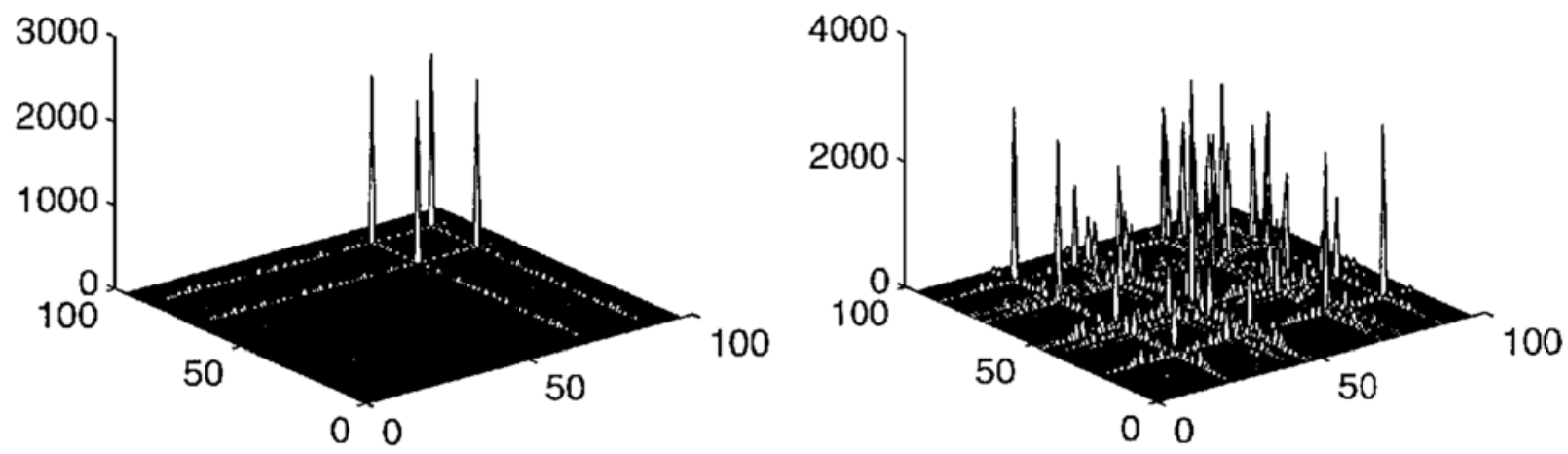

Figure 6. Experimental Update Matrices.

Table 1. Order of Basis Vectors vs. Number of Modes for Experimental Data

\begin{tabular}{lccc}
\hline \hline Number of modes & Vector 198 & Vector 196 & Vector 197 \\
\hline 1 & 1 & 10 & 16 \\
2 & 1 & 11 & 16 \\
3 & 2 & 18 & 25 \\
4 & 2 & 14 & 37 \\
5 & 2 & 14 & 38 \\
\hline \hline
\end{tabular}

Data from Undamaged Structure

The issues from this step in the process include the expected measurement noise and the inevitable lack of a complete measurement set (both spatially and with respect to the critical modes of the structure). As was suggested earlier, this process appears to be fairly robust with respect to noise and the a primary reason to develop this procedure was to attack the incomplete measurement problem. 


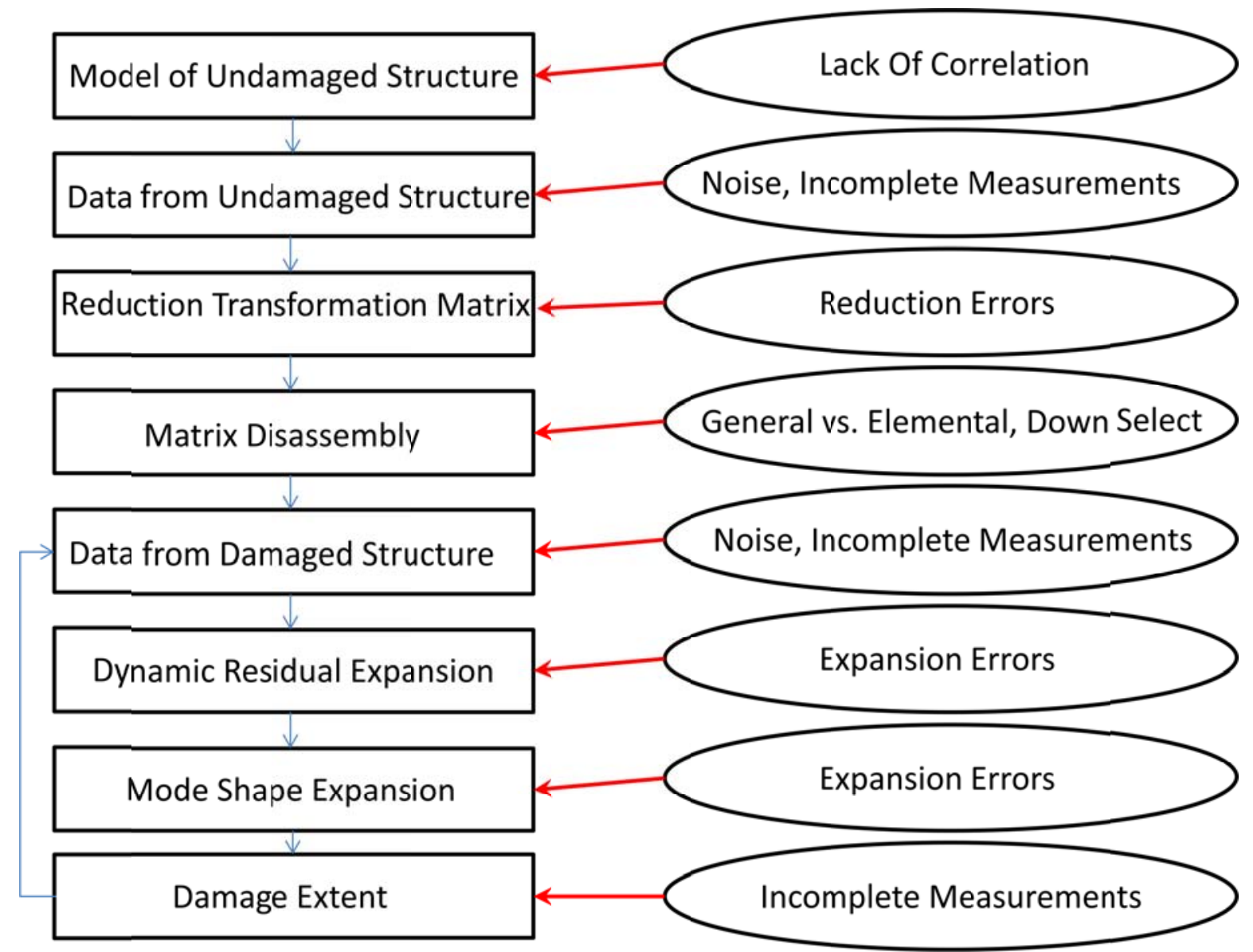

Figure 7. Technical Issues Associated with The Original Procedure.

\section{Reduction Transformation Matrix}

The lack of ability for a reduced system to reproduce the relevant aspects of the full system is a common and well-known problem. The original procedure is likely suffering from reduction errors affecting the system changes that are due to damage through the dynamic residuals and expansion steps that follow. Dealing with the reduction issues is one of the areas of near term work that should be undertaken.

\section{Matrix Disassembly}

The matrix disassembly step proposed in the original process was a general approach that broke the stiffness matrix up into simple springs. This did retain the proper coupling in the end but could allow some non-physical stiffnesses to result. A more rigorous and potentially robust approach would be to disassemble based on the actual element coupling used in building the models. This is certainly technically viable but may be beyond the implemented state-of-the-art for many commercial-scale finite element programs. A second issue at this step involves the need to down select from the huge number of potential simple springs in the disassembled matrix to the set that will be used for creating basis vectors. There is certainly the issue that damage information may be missed or incorrect, however it is technically useful to understand which parts of the structure cannot be monitored for damage given the selected measurement set. There is some additional study that is needed to derive a set of targeted metrics (as opposed to the MAC values) that will help sort the simple springs (or disassembled elements) that will be used for damage monitoring and assessment.

\section{Data from Damaged Structure}

The issues associated with this step are the same as were already mentioned in the Data from Undamaged Structure step. 


\section{Dynamic Residual Expansion}

The issues in this step revolve around the necessary expansion of the limited measured data set to the larger unmeasured data set. Since the inability to measure every Degrees-Of-Freedom (DOFs) in the model is a physical constraint, it is unlikely that much progress can be made on these issues in the near term. However, since this step in the process is critically dependent on the reduction/expansion transformation used, there is some hope for minor near-term improvements that can be realized by improving and tailoring the transformation process used as well as selecting the basis vectors form the disassembly process.

\section{Mode Shape Expansion}

Many of the same issues that plague the dynamic residual expansion exist in this step as well, namely the physical constraint of unmeasured DOFs. In fact the expansion of the mode shapes will be dependent on the expansion of the dynamic residuals in the previous step. Hence, improving and understanding that process is likely to be a precursor to understanding the errors in the mode shape expansion step. Additionally, there is a need to further study the effectiveness of the novel expansion technique provided in the original work.

\section{Damage Extent}

The MRPT-based damage extent based approach follows directly from the expanded residuals and mode shapes produced in the previous steps. However, it has been seen that selection of the proper set of basis vectors for the expansion steps is critical to producing unambiguous results in this step. Hence, the issues associated with this step are largely dependent issues on the problems in implementing the previous steps. This does suggest that the damage extent results will be useful as a metric for the integrated effects of all the previous steps and sensitivity studies involving the application of the entire process are called for as a near term activity.

\section{SUMMARY AND CONCLUSIONS}

The procedure reviewed in this work integrates several technologies into an integrated procedure to perform damage location and extent estimation. Of significant importance is the integration of model order reduction and mode shape expansion into the location and extent algorithm. The end result is a hybrid approach that utilizes an analytical model and experimental data to perform rank constrained damage identification. The procedure utilizes minimum rank perturbation theory, spring disassembly, and Ritz vector calculation to couple these technologies. As a result, the issue of determining damage-affected modes is supplanted by determining a truncation order for a set of localized basis vectors. An application to experimental data in the original work proved to be successful in locating the damage. However, this same application pointed out the need to acquire enough modes to discriminate between the residual basis vectors that drive the rest of the process. Earlier studies suggested that this process is fairly robust with respect to noise. The experimental application also suggested that a sparse analytical model could improve the basis vector selection process.

The technical issues associated with the process reviewed herein need to be systematically addressed by mitigating or understanding the effects. Development work to create targeted reduction techniques, generic disassembly, and basis vector selection processes is needed. Understanding and improving the expansion processes are also called for. Other active sensor sets and damage cases should be exercised to understand the experimental potential and sensitivities of this process more completely.

\section{REFERENCES}

1. Guyan, R. J., "Reduction of Stiffness and Mass Matrices," AIAA Journal, Vol. 3, No. 2, 1965, p. 380.

2. Irons, B.M., "Structural Eigenvalue Problems: Elimination of Unwanted Variables," AIAA Journal, Vol. 3, No. 5, 1965, pp. 961, 962.

3. Kidder, R. L., "Reduction of Structural Frequency Equations," AIAA Journal, Vol. 11, No. 6, 1973 , p. 892.

4. O'Callahan, J., Avitabile, P., and Riemer, R., "System Equivalent Reduction/Expansion Process (SEREP)," Proceedings of the 7th International Modal Analysis Conference (Las Vegas, NV), Society of Experimental Mechanics, Bethel, CT, 1989 , pp. $29-37$.

5. Zimmerman, D. C., Smith, S. W., Kim, H.-M., and Bartkowicz, T., “An Experimental Study of Structural Damage Detection Using Incomplete Measurements," Journal of Vibration and Acoustics, Vol. 118, No. 4, 1996, pp. 543-550.

6. James, G. H., and Zimmerman, D. C., "Reduction/Expansion Studies for Damage Identification of Continuous Aerospace Structures," Proceedings of the 15th International Modal Analysis Conference (Orlando, FL), Society of Experimental Mechanics, Bethel, CT, 1997, pp. 1772-1778. 
7. Imregun, M., and Ewins, D. J., “An Investigation into Mode Shape Expansion Techniques," Proceedings of the 11th International Modal Analysis Conference (Kissimmee, FL), Society of Experimental Mechanics, Bethel,CT, 1993, pp. 168-175.

8. Smith, S. W., Baker, R. J., Kaouk, M., and Zimmerman, D. C., "Mode Shape Expansion for Visualization and Model Correlation," Proceedings of the 9th VPI\&SU Symposium, edited by L. Meirovitch, Virginia Polytechnic Inst. and State Univ., Blacksburg, VA, 1993, pp. 385-396.

9. Levine-West, M., Kissil, A., and Milman, M., "Evaluation of Mode Shape Expansion Techniques on the Micro-Precision Interferometer Truss," Proceedings of the 12th International Modal Analysis Conference (Honolulu, HI), Society of Experimental Mechanics, Bethel, CT, 1994, pp. 212-218.

10. Gafka, G. K., and Zimmerman, D. C., "Structural Damage Detection via Least Squares Dynamic Residual Force Minimization with Quadratic Measurement Error Inequality Constraint," Proceedings of the 14th SEM International Modal Analysis Conference (Dearborn, MI), Society of Experimental Mechanics, Bethel, CT, 1996, pp. 1278-1284.

11. James, G. H., and Zimmerman, D. C., "Utilization of Large Experimental/Analytical Data Sets for Structural Health Monitoring of Aerospace Structures," Proceedings of the 15th International Modal Analysis Conference (Orlando, FL), Society of Experimental Mechanics, Bethel, CT, 1997, pp. 1765-1771.

12. Doebling, S. W., Peterson, L. D., and Alvin, K. F., "Experimental Determination of Local Structural Stiffness by Disassembly of Measured Flexibility Matrices," Journal of Vibration and Acoustics (to be published); see also AIAA Paper 95-1090, April 1995.

13. Doebling, S. W., "Measurement of Structural Flexibility Matrices for Experiments with Incomplete Reciprocity," Ph.D. Dissertation, Aerospace Engineering Sciences Dept., Univ. of Colorado, Boulder, CO, April 1995.

14. Doebling, S. W., "Damage Detection and Model Refinement Using Elemental Stiffness Perturbations with Constrained Connectivity," AIAA Paper 96-1307, April 1996.

15. Robinson, N. A., Peterson, L. D., James, G. H., and Doebling, S. W., "Damage Detection in Aircraft Structures Using Dynamically Measured Static Flexibility Matrices," Proceedings of the 12th International Modal Analysis Conference (Dearborn, MI), Society of Experimental Mechanics, Bethel, CT, 1996, pp. 857-865.

16. Cao, T. T., and Zimmerman, D. C., "A Procedure to Extract Ritz Vectors from Dynamic Testing Data," Proceedings of the 15th International Modal Analysis Conference (Orlando, FL), Society of Experimental Mechanics, Bethel, CT, 1997, pp. 1036-1040.

17. Kaouk,M., "Structural Damage Assessment and Finite Element Model Refinement Using Measured Modal Data," Ph.D. Dissertation, Aerospace Engineering, Mechanics, and Engineering Science Dept., Univ. of Florida, Gainesville, FL, May 1993.

18. Kaouk, M., and Zimmerman, D. C., "Structural Damage Assessment Using a Generalized Minimum Rank Perturbation Theory," AIAA Journal, Vol. 32, No. 4, 1994, pp. 836-842.

19. Simmermacher, T., Zimmerman, D. C., Mayes, R. L., Reese, G. M., and James, G. H., "The Effects of Finite Element Grid Density on Model Correlation and Damage Detection of a Bridge," AIAA Paper 95-1075,April 1995.

20. James, G., Zimmerman, D., and Cao, T., "Development of a Coupled Approach for Structural Damage Detection with Incomplete Measurements", AIAA Paper 97-0362, Proceedings of the 35 $5^{\text {th }}$ Aerospace Sciences Meeting, Reno, NV, January 6-9, 1997.

21. James, G., Zimmerman, D., and Cao, T., "Development of a Coupled Approach for Structural Damage Detection with Incomplete Measurements", AIAA Journal, Vol. 36, No. 12, December, 1998, pp. 2209-2217.

22. James, G. H., Carne, T. G., Hansche, B. D., Mayes, R. L., Reese, G. M., and Simmermacher, T., "Health Monitoring of Operational Structures - Initial Results," AIAA Paper 95-1072, April 1995.

23. Zimmerman, D. C., and Kaouk, M., "Structural Health Assessment Using a Partition and Element Model Update," Journal of the Chinese Society of Mechanical Engineers, Vol. 19, No. 1, 1998, pp. 115-124.

24. Kashangaki, T. A. L., "Ground Vibration Tests of a High Fidelity Truss for Verification of On Orbit Damage Location Techniques," NASA TM-107626, May 1992. 